\title{
System retraining to professional competences of cognitive robots on basis of communicative associative logic of technological thinking
}

\begin{abstract}
There are two main approaches to hardware-software realization of imitative thinking of cognitive robots. First approach is machine learning. Such cognitive robots are used in services industry, for the commercial and entertaining purposes. In article approach to creation of cognitive robots on the basis of modeling of communicative and associative logic of imitative thinking of the person is considered. Cognitive robots on the basis of modeling of communicative and associative logic of imitative thinking of the person are used as lecturers and consultants. Also in professional activity when concepts and competences are strictly defined. The communicative and associative logic of thinking allows to create the symbolical conceiving robot capable to study, realize information requirements (tasks), to train subject areas, to communicate with the help of the speech, to read and write in various languages. The robot on the basis of symbolical language communicative logic solves a problem of the automated imitation of imitative thinking with associative and communicative symbolical language elements of knowledge. The main practical objectives of imitation of imitative thinking are drawing up the intrinsic focused dictionaries of the developed subject domains of knowledge and standard information requirements; drawing up standard procedures of realization of information requirements; formation of networks from communicative and associative symbolical language elements of knowledge of subject domains; expansion of a natural language to functional; creation of systems of speech and text communication in a natural language and recognitions of the speech of interlocutors. In article the system of retraining to professional competences of robots androids is considered on the basis of communicative associative logic of technological thinking by cognitive methods.
\end{abstract}

Keywords: imitative thinking, communicative and associative logic, cognitive professional robot, information requirement
Volume 5 Issue 3 - 2019

\author{
Evgeniy Bryndin \\ Scientific department, Research center \\ ESTESTVOINFORMATIKA, Russia
}

Correspondence: Evgeniy Bryndin, Scientific department, Research center ESTESTVOINFORMATIKA, Novosibirsk, Russia, Email bryndin 15@yandex.ru

Received: April 17, 2019 | Published: May 16, 2019

\section{Introduction}

Imitative thinking represents communicative and associative processes with words, phrases, offers and judgments. At the computer level imitative thinking is imitated by communicative and associative processes with symbolical language elements of knowledge. Imitation of imitative thinking at the computer level is carried out on the basis of symbolical language communicative logic and network communicative and associative representation of symbolical language elements of knowledge. The information circle of the person represents the connected signs of environment of the first alarm level and the language intrinsic environment of the second alarm level. At the first alarm level information on a surrounding reality in the form of signs, signs of situational schemes and situational schemes is stored. At the second alarm level symbolical elements of knowledge are stored in the intrinsic environment: words, phrases, offers, judgments.

The hardware-software information environment of the computer stores symbolical elements of knowledge in coded form with an intrinsic marking supplied with all signs of representatives of a reality and connected signs of situational schemes. To an element of knowledge from the hardware-software environment of the computer there corresponds the element of knowledge from the intrinsic circle of the person connected with the same signs and the scheme of the first alarm level. These elements of knowledge correspond to the same fragments of a reality. As the hardware-software environment works with sign elements of knowledge as language, it is necessary to supply still elements of knowledge of the hardware-software environment with syntactic structures and a language marking according to grammar and rules of a written language to use language practice of the person for understanding of sense of written texts.

The hardware-software symbolical language environment which elements of knowledge have the marking described above and the semantic circle of the expert always correspond to the same surrounding reality or abstraction. Therefore it is possible to imitate symbolical and language thinking of the person at the level of cogitative acts, keeping compliance between contents and language symbolical elements of knowledge, substituting surrounding or virtual (abstract) reality for the language symbolical description with functional the sintaktiky, selecting acts of thought process, keeping the relations between elements of knowledge and representatives realities and developing experience of understanding and transfer of sense.

The person joins semantic acts through the existential, cause and effect relations. Symbolical structures of elements of knowledge are built in memory of the person according to communicative communications of the first alarm level between the corresponding signs of representatives of a reality or abstraction. Symbolical elements of knowledge can be displayed one-to-one in the coded patterns of electric activity of memory of the robot. At such robot semantic acts of the person are substituted for semantic synthesis of the corresponding coded patterns, keeping univocity of structures and 
communication language communications with the corresponding elements of knowledge, it is possible to carry out imitation of symbolical language thinking by means of the robot. The symbolical and language communicative logic of the person is the cornerstone of imitation of symbolical language thinking. Imitation of symbolical language thinking is carried out on communicative and associative communications of elements of knowledge. Therefore it is important to have communicative communications at a stage of representation of knowledge. For this purpose it is necessary to specify subject domain of knowledge, a situation for judgments, the situational moment for offers. Situations connect the situational moments in the semantic focused representation of knowledge of subject domain Representation of knowledge is essential for imitation of symbolical thinking as elements of knowledge and communicative and associative communications between them directly participate in cogitative acts. Elements of knowledge: words, phrases, offers, judgments are stored in memory of the person with communication and associative communications between them, the displaying communications between the corresponding representatives of a reality or abstraction. Symbolical language thinking offers and judgments represents communicative and associative process of realization of information needs of the person. Information requirement is formed in subject domain and is implemented in her system of knowledge. The person gathers experience of realization of information requirements by means of imitative thinking on the basis of cogitative experience of others, accumulating information requirements and their realization in memory. Then, combining the initial information requirements which are in memory person builds new information requirements. The person realizes new information requirement the corresponding combination of realization of initial information requirements, components new. At the person imitative thinking thus develops. If to store realization of information needs for a type of the coded patterns of elements of knowledge in hardware-software memory, then it is possible to automate process of realization of information requirements on communicative and associative logic with use of a functional natural language. ${ }^{1}$

\section{Technology of realization of communicative and associative logic}

Logical unit of communicative and associative logic is the word. The text of information requirement is under construction in a functional natural language of elements of the intrinsic dictionary. The functional natural language contains means of the description of communicative and associative communications between elements of knowledge that allows imitating imitative thinking at the symbolical language level by means of the robot. Communicative and associative language communications between elements of knowledge follow communications of objects, actions, qualities, properties, time, spaces, etc. which also designate elements of knowledge. In language structural, functional and semantic attributes of elements of knowledge are coordinated. Use of words as a part of elements of knowledge of information requirement is set by communicative communications. In language there are grammatical rules of speciation of word forms for generation and expansion of families and generation of communities, communicative rules of formation of phrases, offers of judgments. At each rule the range of definition. For example, at the deductive rule of generalization a range of definition are deductive sets. Deductive rules allow to order the words of language on the generalized sets. The generalized set is presented by convergent sign. Private values of convergent sign are his specification. In language the accent in a word, in the offer, in judgment between offers is fixed sintaktiky. Shock words in the offer are highlighted in bold type. For example, Evgeniy was in China. Evgeniy was in China. Evgeniy was in China. Percussions of a syllable are highlighted in bold type. Communications according to contents of sentences have syntactic designations. On the assembly ascending parse and the morphological analysis selects the offers of information requirement corresponding to acts of thinking and establish grammatical types and signs for each word according to the dictionary of semantic orientation, builds a tree of syntactic submission of words, defines communicative communication of words, phrases and offers. For the analysis the intrinsic dictionary, networks of phrases, offers, judgments, information families and communities are used.

Parse is carried out on a language syntactic marking of information requirement and its structural signs of situational to a marking. By parse are defined, grammatical meanings of elements of knowledge, the words in offers, phrases in offers, offers in judgments, elementary judgments in compound judgments are selected, grammatical types of words, species groups of phrases by grammatical types of words of bases and additional, grammatical types of lexical meanings of information niches of offers, and specific communications of offers of elementary judgments come to light. The morphological analysis is carried out on a language morphological marking and circuit signs of situational. In the course of the morphological analysis morphological values of elements of knowledge and signs of situational schemes of morphological values are defined. Allocation of offers is carried out on syntactic structure in which to words signs and indexes of representatives, grammatical types and lexical meanings are attributed, to phrases semantic values. On the assembly ascending parse and the morphological analysis of texts of information requirement information niches of the offer are defined. The received text after the assembly analysis comes to the system of symbolical and language realization of information requirement-the system of imitation of thinking. Entrance information of system is the information requirement and attributes received in the course of the syntactic and morphological analysis. According to information obtained as a result of the syntactic and morphological analysis of information requirement, the system of imitation of thinking carries out her semantic analysis. At first the type of information requirement is distinguished. Information requirement can be interrogative or incentive. Then by the semantic analysis is defined whether there is a realization of information need for the knowledge base. If there is no her realization, then is defined whether information requirement is standard basic. If information requirement isn't standard basic, then in the course of the analysis is defined whether it consists of standard basic information requirements. If it doesn't consist of standard basic information requirements, then by the semantic analysis is defined whether there is a necessary and sufficient set of procedures of realization in base of abilities both a necessary and sufficient set of elements of knowledge in the knowledge base for her realization. In case as a result of the analysis it becomes clear that in base of abilities and knowledge there are elements for realization of information requirement, then the system of imitation of thinking makes out realization of information requirement from elements of knowledge of information requirement and elements of knowledge for realization from the knowledge base. The system determines indexes on necessary sets of elements of knowledge in the knowledge base in the subject domain set by the user by a marking of the text of information requirement, and on procedures of realization in base of abilities, and defines the scheme of realization according to type and a marking of 
requirement. Attributes of elements of knowledge are specified in a marking: circuit, priznakovy, grammatical (syntactic, morphological), semantic and lexical. Indexes on elements of knowledge and realization are formed through the information receptive field. The information receptive field contains information communications with entrances of network of phrases, offers, judgments and also with realization of standard basic information requirements. It contains instructions about a necessary and sufficient set of procedures of realization for each lexical meaning of information action and about a necessary and sufficient set of elements of knowledge of each procedure of realization and also the information connections with procedures of realization which are stored in base of abilities. Indexes for search of necessary and sufficient set of elements of realization in base of abilities, a necessary and sufficient set of elements of knowledge in the knowledge base, schemes and structures of formation of new phrases, offers, judgments are determined by a marking of information requirement and the field of knowledge set by the user.

The system of imitation of thinking starts the procedure of creation of network of step-by-step realization of information requirement from a necessary and sufficient set of elements of knowledge and procedures of realization. System builds network of step-by-step realization of information needs for knowledge bases and abilities. Then makes an initial marking of elements of knowledge and procedures of realization and their communications. The marking defines signs of participation of words in phrases, signs of situational participation of phrases in offers and situational participation of offers in judgments. After a marking of network of step-by-step realization, it is started on realization of information requirement. Information requirements are considered realized if the syntactic structure of realization, lexical and semantic meanings of components and also procedures of realization are defined. If entrance information requirement is a combination of standard information requirements or their admissible modification, then she realized. Standard incentive information requirement consists of information action and information condition. If information requirement incentive, then is obviously specified in it action. Interrogative information requirement is a question. If information requirement interrogative, then is obviously specified in it the interrogative phrase defining the direction of realization. Realization of interrogative information requirement is based or in network of offers, or in network of judgments. In networks the semantic value of realization of requirement or in the form of the offer, or judgment comes to light. After realization new information requirement is entered in the knowledge base and contacts an element of knowledge which is her realization.

The technology of realization of communicative and associative logic of imitative thinking allows to develop the interactive training systems of natural language level in various objects in educational space. The subject domain of knowledge is presented in the form of communicative and associative network of the information requirements and their realization consisting of elements of knowledge. Communicative phrases form offers, communicative offers form judgments. Between information requirement and its realization communication associative. Realization of information requirement itself can be information requirement. The words of information requirement have the marking specifying or on a lexical meaning, or on the computing procedure, or on the behavioral procedure. For example, the word "put" can be a lexical meaning, either arithmetic action, or behavioral action (to put cubes). For judgments the subject domain and a situation is specified. For offers the situational moment and signs of lexical meanings is specified. Reflection of semantics of subject knowledge is carried out by means of symbolical and language sign system on the basis of communicative and associative logic and in detail signs of situational the relations of entities of objects of a reality and abstraction with elements of knowledge of the intrinsic dictionary. ${ }^{2-7}$

\section{Intrinsic dictionary}

Psyche of the person since the birth in the course of language communication acquires entities in the form of sensual elements of knowledge. The intrinsic dictionary, defining semantic use of morphological words, is necessary for technological thinking of robot, which does not have psyche. Let $\mathrm{S}$ - the spelling dictionary, where $\mathrm{S}=\{\mathrm{Si}\}, \mathrm{Si}$ - a morphological word. The word $\mathrm{Si}$ calls sign of Qij of the representative of Mij from a set of Mi where $\mathrm{Mi}=\{\mathrm{Mij}\}$. Will designate a lexical meaning of the word Si through $\{\mathrm{Mij}, \mathrm{Qij}$, $\mathrm{Si}\}$. Will set communication of lexical meanings of words $\{\mathrm{Si}\}$ with elements of a set Mi set of the sign of relations of Qi where $Q i=\{Q i j$, (Mi, Mij)\}. The set of lexical meanings of the sign of relations with representatives connected by set is the intrinsic dictionary. Words in the dictionary are supplied with sign of indexes according to their sign of relations with representatives. The intrinsic dictionary fixes sign of entities of representatives. The dictionary helps to use words with the lexical meaning and to distinguish representatives whom they call at the symbolical level. Words are used on the basis of sign of indexes. Each sign has three indexes. One index indicates subject domain of knowledge, the second a situation, the third for the situational moment. Words with several lexical meanings, have several sets of indexes. For example, flour and flour. The word the field is used in various subject domains. Each set of indexes defines a lexical meaning of a word.

\section{Subject domains of knowledge}

Subject domains of knowledge represent networks is associative the connected information requirements and their realization from communicatively coherent symbolical language elements (information traces of communicative and associative symbolical and language thinking). Realization of information requirement can be itself information requirement. Then associative network of communicatively coherent elements will be her realization. The combined information requirements consist or of the enclosed information requirements of subject domain of knowledge, or of the sequence of information requirements, or of the sequence of the enclosed information requirements. The combined information requirements consist either from simple, or from simple and combined, or of combined. Information requirements of subject domain of knowledge form the combined expanded network. The set of elements of knowledge with a marking is the knowledge base. Imperative or the question of the knowledge base is information requirement. The set of procedures of realization of information requirement is base of abilities. Information requirement has realization in the knowledge base and base of abilities if there is a necessary and sufficient set of elements of knowledge in the knowledge base and procedures of realization in base of abilities. For the information requirement which is a combination of information requirements for which there is a realization there is a necessary and sufficient set of elements of knowledge in the knowledge base and procedures of realization in base of abilities. The corresponding combination of necessary and sufficient set of the available realization will be realization of the combined information requirement. The symbolical and language communicative logic connects the realized information requirements. 


\section{Algorithmic resolvability communicative associative logic} be set;

Let ID, the intrinsic dictionary of subject domain of knowledge

BIR, the basic information requirements of subject domain of knowledge made of the words of the ID dictionary. Basic information requirement is either the interrogative or imperative offer;

RBIP, realization of basic information requirements;

RCIR, rules of combination of information requirements from basic;

DCANRIR, the designer of communicative and associative network of realization of information requirements of the user;

CANRBIR, communicative and associative network of realization of basic information requirements of subject domain of knowledge.

Let the user it is set of information requirements (IRi), made by the rules RCIR. We will apply to her the designer of DCANRIR. We will receive CANRBIR(i) expansion of CANRBIR network with realization (IRi).

The realization of interrogative information requirement for communicative and associative network of subject domain of knowledge is enabled as follows. Let the offer P1 with a marking be interrogative information requirement with a sing of situational scheme SP1 from the situational scheme S1. The interrogative word in the offer P1 to undescribed sign of Q1 of a lexical meaning of LM1 of information niche of IN1 is known. It is necessary to find in communicative and associative network of subject domain of knowledge the offer $\mathrm{P} 2$ containing phrases and lexical meanings of the offer P1 and the lexical meaning of LM2 which is the description of sign of Q1. The procedure of realization builds according to the offer P1 the P2T template with undescribed sign of Q1 of the offer P2. On the P2T template, his marking, the SP1, S1 procedure of realization looks for the offer P2 in network of communicative phrases of subject domain of knowledge by means of procedures of the morphological, syntactic and semantic analysis. The found offer P2 will be realization. Imperative information requirement for search realization is replaced with equivalent interrogative information requirement. Conclusion shows that the communicative and associative logic is algorithmically solvable.

\section{Modeling of imitative thinking of the cognitive robot}

The technology of realization of communicative and associative logic allows create the symbolical conceiving robot with imitative thinking. Information unit of communication between the robot and the interlocutor is information requirement either speech, or written in natural functional language. ${ }^{7}$ The interlocutor uses information requirements which contain in the knowledge base of the robot. He communicates with the robot by means of combinations of the available information requirements, enriching, thereby, the robot with new information requirements and their realization. The robot receives basic information requirements and their realization during his training. The system of realization of information requirement uses intrinsic dictionaries of subject domains of the knowledge base, the procedure of the analysis, merge (synthesis) and splitting from base of abilities and also network of step-by-step realization of information requirements. The robot realizes information requirement if the knowledge base contains a necessary set of elements of knowledge, the base of abilities contains a necessary set of elements of realization. Imitation of symbolical language thinking is carried out on the basis of symbolical and language communicative logic over symbolical language elements of knowledge of subject domains. Subject domains of knowledge present to network communicatively and is associative coherent symbolical language elements with a sing of situational and a language marking.

\section{Definition}

The network $\mathrm{G}(X t, V t, V t)$ is conceptual representation of knowledge,

$$
\text { If } X t={ }_{x t}^{1} U_{x_{t}}{ }^{2} U_{x_{t}}{ }^{3}
$$

where $x_{t}^{1}$ - a set of tops of judgments with a situational and language marking, a set of tops of the offers having language and situational признаковую a marking: \{ (a lexical meaning, a look) \}, (sign, a niche) $\}, x_{t}^{3}-$ a set of tops of phrases with a sing and language marking, $V t-$ a set of communicative communications of elements of a set of $X t, G t-$ a set of associative communications of elements of a set of $X t, \mathbf{t}$-the discrete moments of emergence of new elements of knowledge, sign - the sign of the representative of a reality indicating the semantic use of a word in the speech and in writing a look - the grammatical characteristic of a set of words which morphological value is formed by the general rules of grammar a niche-uniform situational use of a set of words of different types in various syntactic sentence structures (the niche can be subject and object, procedural, a circumstance, attributive, additional. Associative communications connect tops which are information requirement and its realization. Communicative communications proceed from tops of communicative elements of knowledge in the general top. Realization of information requirement is formed by procedures of the semantic syntactic and morphological analysis, merge and splitting of elements of information need of the user, the words of the intrinsic dictionary and elements of knowledge of subject domain and procedures of realization of information actions of information requirement. Procedures contain in base of abilities.

The robot imitates imitative thinking. On new information requirements the robot issues the version of realization if it enough available communicative and associative elements of knowledge and procedures has realization. If information requirement or realization of information requirement contains information action, then the standard procedure of realization is started. The reference to the procedure of realization is specified in a marking of information action. For example, let imperative information requirement "is set to find the sum the $\mathrm{X}$ plus of $\mathrm{Y}$, at $\mathrm{X}$ is equal $15, \mathrm{Y}$ is equal to 10 ". " $\mathrm{X}$ it is equal 15, Y ravno10" is information condition. Communicative phrases "X It Is Equal" and "equally 15" in information condition of information action "plus" initiates (Figure 1). The compulsory provision of feasibility of information requirement is completeness of the knowledge base and base of abilities, that is existence of necessary and sufficient set of elements of knowledge and abilities of crossdisciplinary subject domain for training in a healthy lifestyle (Figure 2).

The healthy lifestyle of the person is under construction on the basis of high-quality regularities. High-quality regularities are described by causes and effect relationships. The communicative and associative network of knowledge of the cross-disciplinary 
subject domain concerning a healthy lifestyle contains causes and effect relationships between elements of knowledge describing useful effects and the corresponding improving investigations generated by them. The realization of a healthy lifestyle is enabled by useful habits. Purity of an organism is reached by a habit of daily hygiene. Consultation of the robot in communicative and associative network of elements of knowledge of subject domain is based as a chain of relationships of cause and effect. For example, in the mode short consultation: (a habitaily hygiene)-(purity of an organism). Realization of information requirement can be information requirement. In this case on a communicative tree of lexical meanings of the reason by their synthesis we reach associative top. Then we determine realization by associative communication. The robot can train along with consultations in physical exercises. ${ }^{4}$ To physical culture for normalization of a tone of an organism. To gymnastics for normalization of rhythms of functioning of an organism. To charging on normalization of a power system. Realization of information requirement can be information requirement. In this case we determine realization by associative communication "A formulation of Pythagorean theorem". For example, let imperative information requirement "Is set to formulate Pythagorean theorem". On a lexical meaning of the word "formulation" we find through the intrinsic dictionary a representative phrase "wording of the theorem" in network of the subject domain of knowledge specified in a marking of information requirement. Then on a tree of communicative phrases with lexical meanings of information requirement by their synthesis we reach associative top "a formulation of Pythagorean theorem". Further on associative communication from associative top of information requirement we pass to associative top of realization "the square of a hypotenuse is equal to the sum of squares of legs. The robot with communicative and associative logic of imitative thinking, having remote communication with the person, is capable to carry out cognitive information needs of the person for many spheres of activity. ${ }^{8-15}$

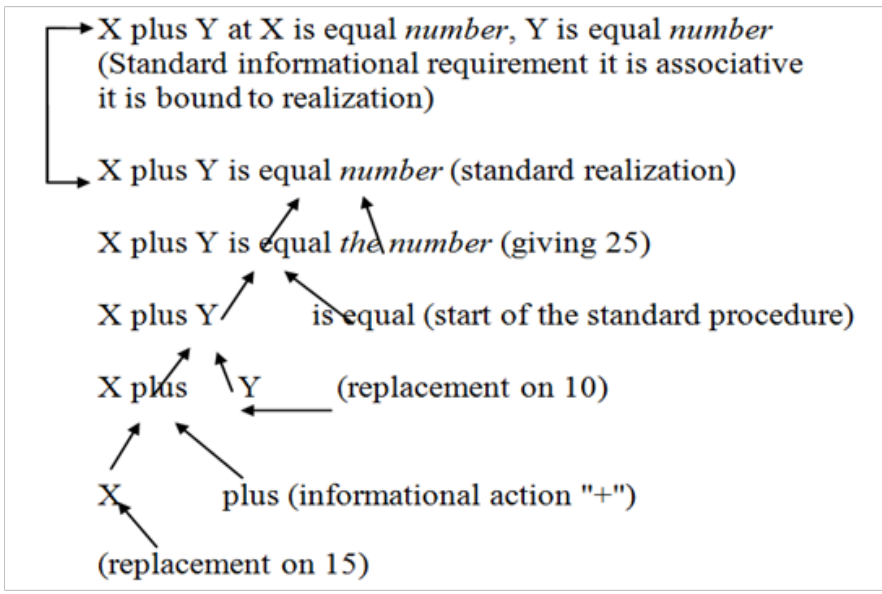

Figure I Network structure of addition.

\section{System of realization of information requirement}

The system of realization of information requirement contains the knowledge base, base of abilities, behavior model, environment model, programmable mekhatronny, navigation and other devices of realization of adaptive technological behavior. The knowledge base supports associative communicative network of information requirements and their realization. The base of abilities contains procedures of realization of technological thinking and adaptive technological behavior. Realization of information requirement undertakes or from the knowledge base, or is developed by a standard procedure of realization of base of abilities for the current information requirement (Figure 4), or the network of step-by-step realization on the combined information requirement is formed (Figure 5).

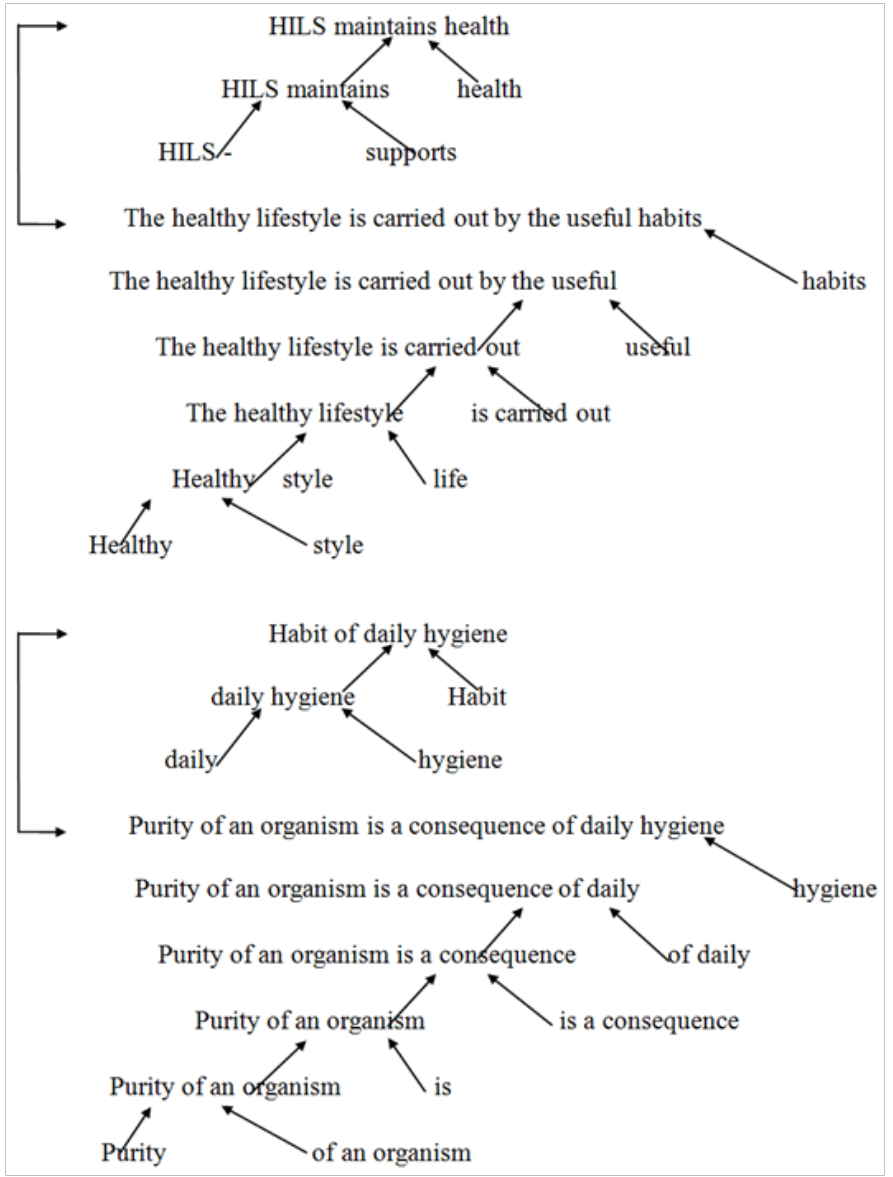

Figure 2 Communicative-associative network of healthy lifestyle.

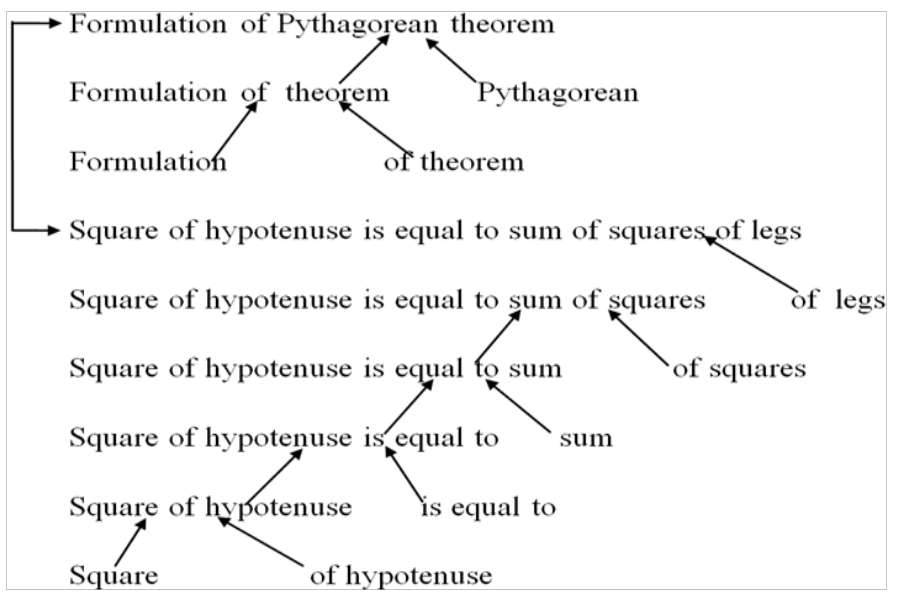

Figure 3 Associative communicative network of Pythagorean Theorem. 


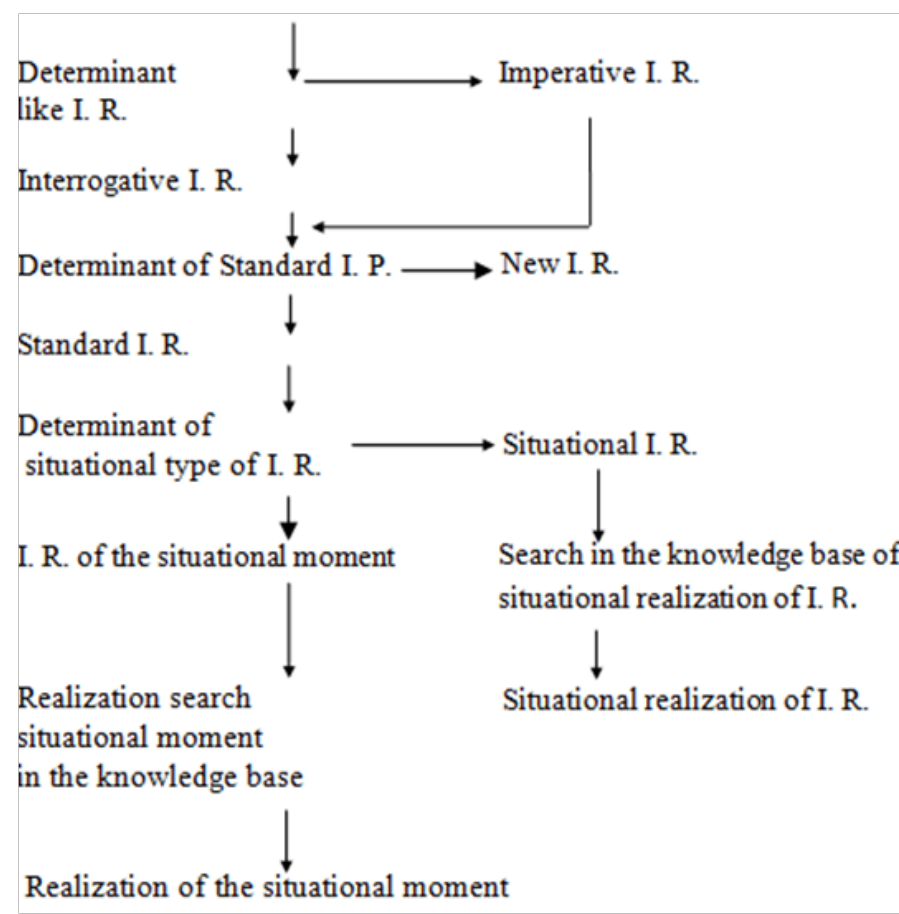

Figure 4 Realization of standard information requirement (I. R.).

Determinant of admissible set
communicative elements
in the knowledge base and base
abilities for I. R. realization.
$\begin{aligned} & \text { Creation of network step-by-step } \\ & \text { realization of I. R. on her } \\ & \text { to functional marking }\end{aligned}$

Figure 5 Realization of new information requirement (I. R.)

\section{Functional structure of the cognitive professional robot with retraining}

The functional structure of the cognitive professional robot with retraining consists of various systems with artificial intelligence (Figure 6).

The robot with symbolical language thinking has system of recognition of the interlocutor, system of speech input of information requirements, system of realization of information requirements (system of imitation of imitative thinking), network system of synthesis of the speech in the text of realization of information requirement. ${ }^{4-15}$ The system of realization of information requirement contains system of assimilation of knowledge, system of symbolical and language communication, system of training, base of knowledge, abilities base, the neural network system of reading printing system and system of graphic display. The system of training contains subsystems of machine translation. The system of recognition of interlocutors is neural network system. Information unit of communication between the robot and the interlocutor is information requirement. The interlocutor uses information requirements which contain in the knowledge base of the robot. He communicates with the robot by means of combinations of information requirements, enriching, thereby, the robot with information requirements. The robot receives new basic information requirements, elements of knowledge and realization during his training.

Acquaintance of the robot to the person is carried out through neural network system of face recognition. If the person is unknown to the robot, then the receptive system remembers his speech dictionary and the person. If he is known to the robot, then the system will customize system of speech input of information requirement on the speech dictionary of the interlocutor. After that information contact between the robot and the person begins. The system of speech input will transform speech information requirement to the text in a functional natural language. Specialization of cognitive adaptive robots is carried out on the basis of knowledge bases, bases of abilities and implementers of behavior. Cognitive adaptive robots with imitative thinking and adaptive behavior have prospect of broad practical application.

\section{Cognitive methods structuring, classification identification of knowledge by logical formats}

Process of cognitive search of regularities consists of two stages: Cognitive analysis of knowledge; identification of regularities by forecasting methods by results of the analysis. The cognitive analysis of data uses mathematical methods and algorithms, the systems of data processing and technology of visual representation of data.

Cognitive methods of the analysis of data: Statistical methods, methods of computer mathematics, optimizing methods, expert methods, synergetic methods, methods of indistinct sets, methods of fractal mathematics, methods of conflict situations.

Algorithmic systems of data processing: Subject-oriented analytical systems, the systems of the statistical analysis, the trained neural networks, associations on analogies, trees of decisions, evolutionary programming, algorithms of search, the system of visualization of multidimensional data.

Methods of the forecast of situations: The determined forecast, the statistical forecast, a method of program forecasting, a method of heuristic forecasting, temporary ranks, extrapolation method, expert method, the forecast on the basis of linear regression, the interpreted method, the case analysis, the synergetic analysis, the evolutionary statistical forecast.

The tasks solved by cognitive methods of the analysis of data: Detection and assessment of the hidden regularities, detection and assessment of influence of the hidden factors, assessment of the current situation, the forecast of development of the situation, formation and optimization of the operating decisions.

\section{Data processing languages}

Language of statistical researches R contains a wide range of various functions (temporary ranks, forecasting, classification, clustering) and allows carry out profound analytics: identification of the facts, entities 
or objects; to define subject; to carry out classification. The Python language contains means of statistical modeling and processing of big data flows. The MATLAB language contains means of processing of stream information, the analysis of the obtained and predicted data in the field of scientific research.

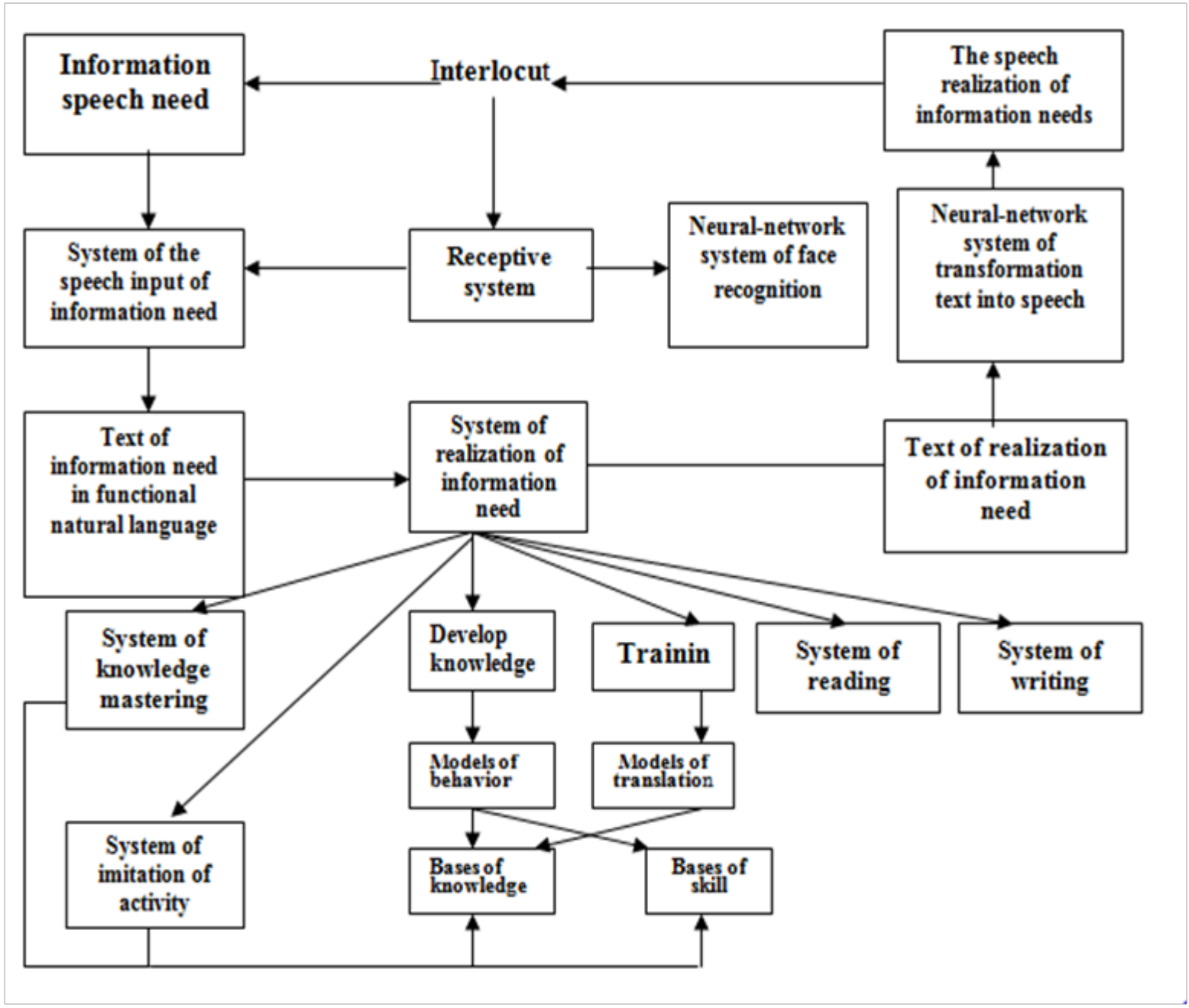

Figure 6 Functional structure of the cognitive professional robot.

\section{Digital systems of modeling}

Deductor the analytical platform developed by the Base Group Labs company. The most demanded analysis algorithms (trees of decisions, neural networks, the self-organized cards, etc.) are built in Deductor, there are tens of ways of visualization and integration into a set of sources/receivers of data is provided. In system technologies which on the basis of uniform architecture allow pass all stages of creation of the analytical platform are applied: from creation of storage of the models given before automatic selection and visualization of the received results. SAS Enterprise Miner is the software product developed for the purpose of creation of exact predictive and descriptive models on the basis of large volumes of information. The software of STATISTICA uses algorithms of access to data, their transformations and creation of predictive models.

\section{Cognitive technology of search of regularities}

The machine technology at which the computer analyzes experimental data and writes own program of detection of statistical regularities is cognitive technology. The cognitive machine technology helps to take new regularities effectively. Mathematicians of Deep Mind company have developed an algorithm of machine learning IMPALA which allows separate parts of system to study performance of several tasks at once, and then to exchange data among themselves. ${ }^{16}$ On the basis of similar algorithms, temporary ranks and the systems of modeling Deductor, SAS Enterprise Miner and STATISTICA it is possible to receive set of the regularities and data sufficient for creation of models of nanostructures with necessary parametrical and structural resonant properties.

\section{Conclusion}

The cognitive robots with communicative and associative logic of thinking having the systems of machine retraining of realization of information requirements will be able quickly to change professional qualification and competences. The international scientific and engineering society gradually moves to technical realization of the cognitive professional robot with retraining. The automated system of retraining can retrain the cognitive robot on other specialization. 
The expert has to prepare the knowledge base, base of abilities, behavior model and model of the environment. Degree of accuracy of the description of the external environment, behavior model and realization of information requirement defines correctness of performance of human tasks by robot.

In the future in labor market cognitive robots with retraining will perform professional works, and the person will occupy a niche of scientific research of creative innovative activity. Robots become independent subjects of social environment. Social cognitive smart robots are used as guide, seller, lecturer, vacuum cleaner, nurse, volunteer, security guard, administrator of hotel.

\section{Acknowledgments}

None.

\section{Conflicts of interest}

The author declares there is no conflict of interest.

\section{References}

1. Evgeniy G Bryndin. The Robot with imitative thinking. PNIPU bulletin: Electrical equipment, Information technologies. Control systems. 2015. p. 5-36.

2. David Vernon. Artificial Cognitive Systems. UK: MIT Press; 2014. 70 p.

3. Hooman Samani. Cognitive Robotics. USA: CRC Press; 2015. 220 p.

4. Evgeniy G Bryndin, Cognitive robot consultant for a healthy lifestyle. III International scientific conference "Information Technologies in Science, Management, the Social Sphere and Medicine”. 2016. p. 484-488. 2016.

5. Bryndin EG. Cognitive robots. Management of development of largescale systems. 206. p. 285-294.
6. Evgeniy Bryndin. Cognitive Robots with Imitative Thinking for Digital Libraries, Banks, Universities and Smart Factories. International Journal of Management and Fuzzy Systems. 2017;3(5):57- 66.

7. Evgeniy Bryndin. Technological Thinking, Communication and Behavior of Androids. Communications. 2018;6(1):13-19.

8. Stefan Wermter, Günther Palm, Mark Elshaw. Biomimetic Neural Learning for Intelligent Robots: Intelligent Systems, Cognitive Robotics, and Neuroscience. 2005. 389 p.

9. Ahmed S Al-Araji, Khulood E Dagher. Cognitive Neural Controller for Mobile Robot. IJCCCE. 2015;15(1):46-60.

10. Evgeniy Bryndin. Program Hierarchical Realization of Adaptation Behavior of the Cognitive Mobile Robot with Imitative Thinking. International Journal of Engineering Management. 2017;1(4):74-79.

11. Evgeniy Bryndin. Technological, Economic and Social Aspects of Management by Development of the Digital Industry 4.0. International Journal of Managerial Studies and Research (IJMSR). 2018;6():19-30.

12. Evgeniy Bryndin. Directions of Development of Industry 4.0, Digital Technology and Social Economy. American Journal of Information Science and Technology. 2018;2(1):9-17.

13. Evgeniy Bryndin. Social Cognitive Smart Robots: Guide, Seller, Lecturer Vacuum Cleaner, Nurse, Volunteer, Security Guard, Administrator. Communications. 2019;7(1):6-12.

14. Evgeniy Bryndin. Cognitive smart robots with technological thinking and behavior for industry and social sphere. Science, Technology and Life: Proceedings of articles the V International scientific conference. 2019.

15. Evgeniy Bryndin. Digital technologies of the industry. USA: Nova Science Publisher; 2019. 252 p.

16. Lasse Espeholt, Hubert Soyer, Remi Munos, et al. IMPALA: Scalable Distributed Deep-RL with Importance Weighted Actor-Learner Architectures. Computer Science (Machine Learning). 2018. 